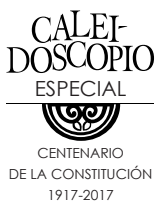

\title{
La Constitución mexicana: primera carta social en el orbe. Cien años de reformas y adiciones entre el cambio y la deformación
}

The Mexican Constitution, first social charter in the world. One hundred years of reforms and additions between change and deformation

CLAUDIO ANTONIO GRANADOS MACÍAS'

RESUMEN

En el presente artículo se analiza si la Constitución de 1917 es la misma a cien años de su expedición, por lo que se ha estudiado su creación, contenido, legitimidad, esencia y evolución. El resultado de confrontar la Constitución revolucionaria con la actual arroja un proceso transformador y deformador a través de 707 enmiendas efectuadas en el periodo de estudio, que nos permite afirmar que la esencia social de la Carta Magna continúa ahí, pero que requiere ser sometida a un proceso de revisión integral para reescribir sus contenidos y devolverle la sistematización y coherencia de su redacción original.

Palabras clave: Constitución de 1917, reformas, evolución.

ABSTRACT

This article aims to analyze if the Constitution of 1917 is the same one hundred years after its issuance, therefore its creation, content, legitimacy, essence and evolution have been studied. The result of con-

1 Universidad Autónoma de Aguascalientes. 
fronting the revolutionary Constitution with the current one, produce a transforming and deforming process through 707 reforms made to the Fundamental Law, which allows us to say that the social essence of the Magna Carta continues there, but that it requires submission to a process of integral revision, to rewrite its contents and give back the systematization and coherence of its original wording.

Keywords: Constitution of 1917, reforms, evolution.

A cien años de la expedición de la Constitución Política de los Estados Unidos Mexicanos, son diversas las voces de especialistas, entre ellos Héctor Fix-Fierro y Diego Valadés (2017), que creen necesario hacer adecuaciones a la Ley Fundamental para retirar de sus contenidos la excesiva reglamentación que ha terminado por deformarla, y así darle coherencia como sucedía en el texto original.

Giovanni Sartori, con motivo del arribo a la presidencia de la República de Vicente Fox en el año 2000, señaló que podía proponerse y aprobarse una reforma constitucional importante, debido al entusiasmo que se vivía por escribir una nueva Constitución; sin embargo, apuntó que en su experiencia, las nuevas constituciones son muy difíciles de escribir, y generalmente son un desastre debido a que los representantes populares que las escriben son malos creadores de constituciones. Además, la Constitución mexicana era una buena Constitución cuando se expidió, donde la mayoría de sus textos eran considerados inteligentes y apropiados, sugiriendo no caer en el sueño de escribir una nueva Constitución: "nunca lo conseguirán, y si lo consiguen será horrible". El profesor Sartori remarcó que todas las nuevas constituciones, las más recientes, son hechas por asambleas constituyentes, y éstas no pueden redactar una Constitución. Todas las normas supremas fueron hechas por expertos, por constitucionalistas y aprobadas subsecuentemente por el parlamento, era aquella una buena manera de hacer una Constitución. En el año 2012, Sartori seguía creyendo que la Carta Magna mexicana era buena y que sólo debía ser reformada para remarcar su sentido democrático (Islas, 2012).

Otros actores, como Bernardo Bátiz, han señalado que el país necesita una nueva Carta Magna porque la que nos rige de 1917 ha tenido muchos cambios y ha sido prácticamente transformada. Lorenzo Meyer advirtió que pese a la situación que se vive en México de división social, no existen las condiciones históricas y políticas para elaborar una 
nueva Constitución, porque -agregó- si bien, de facto, hoy uno se le pude imponer a otro, en el plano constitucional todavía no (López, 2017).

De lo anterior, el problema que resulta es la cuestión que plantea si es necesario que se expida una nueva Constitución, o bien, siguiendo la opinión de una mayoría de juristas y estudiosos, sólo se requiere emprender un proyecto de reformas que se circunscriban a reescribir el texto fundamental para reordenarlo, para hacerlo sistemático, para suprimir las contradicciones, superar sus lagunas, mejorar su redacción; haciendo una recapitulación con relación a su texto y darle al país -a la sociedad- una Constitución legible que sea para los ciudadanos.

La exposición nos lleva a otros cuestionamientos que implican conocer: ¿cómo era el México cuando la Constitución fue elaborada?, ¿cómo es la nación hoy?, ¿cómo esperamos que sea en el futuro? Consideramos pertinente aplicar el método comparado e histórico para encontrar las respuestas que buscamos. Se aborda en este trabajo el contexto desde el cual se convoca al Poder Constituyente, observando cómo se da su integración, el resultado obtenido con la expedición de la Constitución y las opiniones que de la norma fundamental emitieron los juristas, actores políticos y sociales de la época. Luego, confrontamos los textos original y actual de la Constitución para observar el proceso de transformación y cambio de sus contenidos, presentando un breve cuadro donde se sistematizan las reformas sufridas hasta el 15 de septiembre de 2017 (última fecha de su reforma), enmiendas que se localizarán y ordenarán compulsándolas con los registros del Diario Oficial de la Federación.

El objeto del artículo es desentrañar qué ha pasado con nuestra norma suprema a cien años de iniciada su vigencia. Como objetivos específicos analizamos si la Carta Magna sigue conservando su esencia, ¿se mantienen aún sus decisiones políticas fundamentales o estamos frente a una nueva Ley Fundamental?; si las reformas y adiciones a su texto son justificables; ¿qué podemos modificar en la Constitución y qué no?; ¿la Constitución hoy es coherente?, ¿o estamos frente a un texto enmendado en tal forma que ha deformado su integridad?; finalmente, ¿qué requiere la Constitución a cien años de vigencia?

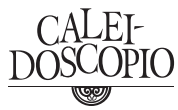


DE LA ASAMBLEA CONSTITUYENTE A LA EXPEDICIÓN DE LA PRIMERA CONSTITUCIÓN SOCIAL DEL ORBE

La primera ocasión que Venustiano Carranza manifestó su propósito de convocar una asamblea constituyente, aparece en un cable dirigido a su representante en Washington, Eliseo Arredondo, fechado el 3 de febrero de 1915 desde Veracruz, que dice: "Cuando la paz se restablezca convocaré Congreso debidamente electo por todos los ciudadanos el cual tendrá características de Constituyente, para elevar a preceptos constitucionales las reformas dictadas durante la lucha" (Rabasa, 1997: 91).

Parece que Carranza no tenía del todo claro el fin de reunir un poder constituyente, ni el significado y alcance de convocarlo, pues todo indica que su interés estaba más alineado a congregar una asamblea legislativa que adoptara facultades de "Poder Revisor de la Constitución", para elevar a rango constitucional las leyes expedidas por los constitucionalistas en 1915, así como integrar en el texto fundamental el programa propuesto en su Plan de Guadalupe y otros planes, así como proclamas revolucionarias, con el propósito de ganarse a la ciudadanía en demérito de sus opositores Villa y Zapata.

Carranza, entonces, adoptó una atinada estrategia sociopolítica: evitar que los convencionalistas consiguieran el respaldo de los elementos populares del país, adoptó sus propuestas agrarias, obreras (Garciadiego y Kuntz, 2011: 559) y ordenó, durante el bienio 19151916, una intensa campaña de propaganda en la prensa nacional y extranjera. Participaron en esa campaña Félix F. Palavicini, en "Un nuevo Congreso Constituyente"; Manuel Aguirre Berlanga, en "Reformas a la Constitución"; y Antonio Manero, en "Por el honor y por la gloria" (Rabasa, 1997: 91). Palavicini (1998: 28), en su publicación, ya estructuraba el camino que Carranza tomaría para establecer al Constituyente, señalando:

El Jefe del movimiento constitucionalista lo es también de la Revolución social, y como esta última, atendiendo a las exigencias del pueblo, ha venido procurando y haciendo realidad trascendentales reformas, reformas que dentro del funcionamiento de los poderes legales, no tendrían justificación, si no es la de que la ley suprema es la salud del pueblo, ocurre desde luego la pertinente observación de que será preciso garantizar la estabilidad de estas reformas, su depuración y perfeccionamiento por me- 
dio de un poder legislativo, que siendo de origen popular, tenga natural y jurídicamente soberanía.

El 14 de septiembre de 1916, Venustiano Carranza expidió el decreto de reforma a los artículos $4^{\circ}, 5^{\circ}$ y $6^{\circ}$ del decreto del 12 de diciembre de 1914, que había adicionado al Plan de Guadalupe, donde se anunciaba la convocatoria del Constituyente. La exposición de motivos de la convocatoria aclaraba que las reformas que se propondrían en el proyecto tocarían a la organización y funcionamiento de los poderes públicos, las que no podían o debían alcanzarse a través de los "trámites" establecidos en la Constitución de 1857 (Tena, 1998b: 627)² y, finalmente, invocando la soberanía popular de acuerdo al artículo 39 de la Ley Fundamental entonces vigente; era preciso reunir una asamblea constituyente. Por su importancia y en atención al interés de nuestro trabajo, reproduciremos el texto del artículo $4^{\circ}$ del decreto:

Artículo $4^{\circ}$. Habiendo triunfado la causa constitucionalista y estando hechas las elecciones de Ayuntamientos en toda la República, el Primer Jefe del Ejecutivo Constitucionalista, Encargado del Poder Ejecutivo de la Unión, convocará a elecciones para un Congreso Constituyente, fijando en la convocatoria de la fecha y los términos en que habrá de celebrarse y el lugar en que el Congreso habrá de reunirse.

Para formar el Congreso Constituyente, el Distrito Federal y cada Estado o Territorio nombrarán un diputado propietario y un suplente por cada sesenta mil habitantes o fracción que pase de veinte mil, teniendo en cuenta el censo general de la República de 1910 (INEGI, 1910). ${ }^{3}$ La población del Estado o Territorio que fuere menor de la cifra que se ha fijado en esta disposición, elegirá, sin embargo, un diputado propietario y un suplente.

2 El artículo 127 de la Constitución liberal de 1857 señalaba sobre la reforma constitucional lo siguiente: "La presente Constitución puede ser adicionada o reformada. Para que las adiciones o reformas lleguen a ser parte de la Constitución, se requiere que el Congreso de la Unión, por el voto de las dos terceras partes de sus individuos presentes, acuerde las reformas o adiciones, y que éstas sean aprobadas por la mayoría de las legislaturas de los Estados. El Congreso de la Unión hará el cómputo de los votos de las legislaturas y la declaración de haber sido aprobadas las adiciones o reformas".

315.2 millones de habitantes.

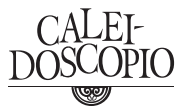


Para ser electo diputado al Congreso Constituyente, se necesitan los mismos requisitos exigidos por la Constitución de 1857 (Tena, 1998b: 615) ${ }^{4}$ para ser diputado al Congreso de la Unión; pero no podrán ser electos, además de los individuos que tuvieren los impedimentos que establece la expresada Constitución, los que hubieren ayudado con las armas o sirviendo en empleos públicos a los gobiernos o facciones hostiles a la causa constitucionalista (Rabasa, 1997: 92).

El párrafo tercero del artículo $4^{\circ}$ del decreto que reforma el Plan de Guadalupe, publicado el 14 de septiembre de 1916, arriba citado, contiene uno de los elementos que determinaron el problema de "legitimidad" del Congreso Constituyente de Querétaro, y que, por consecuencia, puso en entredicho la validez del Código Supremo de 1917. En efecto, la prohibición que estableció señalaba: "no podrán ser electos, además de los individuos que tuvieren los impedimentos que establece la expresada Constitución, los que hubieren ayudado con las armas o sirviendo en empleos públicos a los gobiernos o facciones hostiles a la causa constitucionalista" (Rabasa, 1997: 92).

Es importante enmarcar que se entiende por "poder constituyente": el fundamental y originario poder de los individuos de decidir sobre la forma y sobre el rumbo de la asociación política del Estado (Fioravanti, 2003: 41). Dos son las características que Sieyès atribuía al poder constituyente: a) es un poder originario y único, que no puede encontrar su fundamento fuera de sí mismo porque más allá de él no hay nada; b) es un poder sin condiciones frente al cual no existen límites, ni de tipo formal ni de carácter material. En principio, el poder constituyente lo puede querer todo y quererlo de la forma en que le parezca oportuno (Mora, 2002: 26-27).

El poder constituyente no puede ser definido jurídicamente en términos de constitucionalidad o inconstitucionalidad, o de legalidad o ilegalidad; si puede y debe ser definido políticamente, que sea en términos de legitimidad. Esto es, el poder constituyente tiene que ser un poder legítimo, un poder susceptible de ser explicado en términos racionales, aceptado por la sociedad, y tiene que ser así porque

4 "Art. 56. Para ser diputado se requiere: ser ciudadano mexicano en ejercicio de sus derechos; tener veinte y cinco años cumplidos el día de la apertura de las sesiones; ser vecino del Estado o Territorio que hace la elección; y no pertenecer al estado eclesiástico. La vecindad no se pierde por ausencia en desempeño de cargo público de elección popular". 
la Constitución es la expresión de un orden igualitario y libre (Mora, 2002: 27-28).

La línea que separa a un poder constituyente legítimo de otro ilegítimo es, necesariamente, imprecisa. Por eso, la teoría del poder constituyente se ha centrado en identificar lo que podríamos definir como señales características de la legitimidad, que nos permitan determinar cuándo un poder constituyente puede ser calificado como legítimo y cuándo no. Tales señales hacen referencia a dos elementos importantes: a) la titularidad del poder constituyente, y b) el proceso a través del cual este poder debe expresarse (Mora, 2002: 28).

En el caso que nos ocupa, la titularidad del Constituyente de 1916-1917 recayó en personajes que eran considerados aptos para el régimen carrancista, excluyendo a los opositores al bando constitucionalista, lo que enmarcó la ilegitimidad de origen de la asamblea reunida en Querétaro. El problema fue estudiado y solventado por la doctrina, señalando que la Constitución fue obra de una asamblea constituyente ad hoc, producto de una verdadera revolución, que por serlo, modifica en forma violenta los fundamentos constitucionales de un Estado, redactando un nuevo Código Supremo que, en palabras de Guastini (2001: 40), es un producto extra ordinem, privado de fundamento de validez en normas propias del ordenamiento constitucional precedente.

El ministro Felipe Tena Ramírez (1998a) refería que una Constitución es legítima cuando la fuerza y la autoridad del poder constituyente en que descansa su decisión es reconocida, y citaba sobre la legitimidad de la asamblea de Querétaro:

El modo normal de comprobar la aceptación de una Constitución emanada de una revolución consiste en apelar al pueblo ad referendum; la Constitución así admitida es una Constitución ratificada. Las condiciones que prevalecían en 1917 impedían emplear ese procedimiento democrático. La revolución triunfante se había dividido en varias facciones; una de ellas, la carrancista, que fue la que reunió al Constituyente, hallábase en situación precaria respecto a las demás, y al alcanzar la victoria por medio de las armas, jamás pensó jugársela en los comicios.

En 1917, y durante los años que inmediatamente le siguieron, las ideas avanzadas de la Constitución pertenecían a una minoría; una decisión democrática les hubiera sido desfavorable. Hay, pues, que convenir en que la Constitución de 17 fue en sus orígenes una Constitución impuesta.

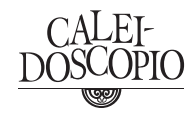


Pero más tarde la paz se organizó de acuerdo con esa Constitución; su vigencia nadie la discute, sus preceptos están en la base de toda nuestra estructura jurídica y son invocados por todos para justificar o para combatir los actos de los gobernantes. La Constitución impuesta ha sido, de ese modo, ratificada tácitamente por el pueblo mexicano y reconocida como su ley suprema por los países extranjeros. "Cuando el poder que al fin logra mantenerse es estimado como Derecho, y muere paulatinamente el Derecho que no se consolida, vuelve a producirse nuevamente la unidad" (Tena, 1998: 73-74).

El Congreso Constituyente reunido en Querétaro se integró con 59 abogados, 33 militares, 21 médicos, 18 ingenieros, 15 profesores, 8 periodistas, 8 campesinos, 6 comerciantes y 4 obreros. La diversidad de los constituyentes deja ver la transición de un México predominantemente rural a un país moderno con obreros y profesionistas (Galeana, 2016: 12-13).

Finalmente, los objetivos del proyecto de reformas a la Constitución de 1857 que Carranza presentó ante el Congreso Constituyente eran: reducir del texto fundamental lo que lo hacía inaplicable, suplir sus deficiencias y, sobre todo, limpiarlo de todas las reformas que no hayan sido inspiradas más que en la idea de poderse servir de ella para entronizar la dictadura; sin embargo, el proyecto no tendría el fin que esperaba. La asamblea de Querétaro rechazó expresamente el guion que Carranza había promovido; planteaba, en cambio, una versión alterna de la dictadura porfirista, en la que la Revolución no se entendía simplemente como una rebelión contra el despotismo político, sino también como una lucha en favor de la reforma agraria y social. De acuerdo con esta interpretación, a Carranza no le correspondía el papel de líder restaurador de las libertades y derechos de los ciudadanos mexicanos, sino el de viejo reaccionario que se opuso a las reformas que exigía la Revolución. Así, la primera interpretación de la Constitución de 1917 insistió en que el constitucionalismo social se había logrado en el Congreso Constituyente, a pesar de la oposición del primer jefe y sus diputados. Los principales autores que argumentaron que la Constitución se había redactado en contra de los deseos del primer jefe fueron los constituyentes vinculados a Álvaro Obregón. Por ejemplo, Juan de Dios Bojórquez argumentó que en el Congreso Constituyente se enfrentaron dos partidos políticos: por un lado, "los hombres del 'apostolado', grupo de venerables diputados cuyo mérito 
era el decirse amigos íntimos de don Venustiano"; y por otro, el grupo de "izquierdas", constituido, como se decía, por "los constitucionalistas de abolengo": jóvenes militares, obreros y maestros comprometidos con los ideales de la Revolución. En contraste con las versiones de Bojórquez, los constituyentes carrancistas, como Pastor Rouaix y Félix Palavicini, insistieron en que Carranza no interfirió en los debates constituyentes ni se opuso al radicalismo constitucional (Andrews, 2017: 95-96).

El trabajo más notable del Constituyente fueron los artículos de relevante contenido social: $3^{\circ}, 27,123$ y 130 . El artículo $3^{\circ}$, propuesto por Carranza, hacía referencia a la plena libertad de enseñanza en lo general, pero laica, tratándose de los establecimientos públicos de educación, sin embargo, la asamblea lo amplió para excluir a las corporaciones religiosas de la instrucción primaria y declaró gratuita la educación en los establecimientos oficiales. La discusión de este artículo atrajo la presencia del primer jefe, que ya no habría de concurrir después a ninguna otra sesión (Rabasa, 1997: 100).

Artículo 123. La materia laboral estaba agotada en el artículo $5^{\circ}$, el cual fue rechazado como se encontraba originalmente, continuándose el debate en varias sesiones hasta el momento en que se presentó la propuesta relativa a que todas las cuestiones obreras estuvieran insertas en un artículo especial, para lo cual se sometió a consideración de la asamblea un proyecto de bases en materia laboral. Cravioto y Manjarrez son los responsables del establecimiento del título sexto dedicado al trabajo y la previsión social, que contuvo las más avanzadas normas para su época en beneficio de los trabajadores. Así, la Constitución de 1917 fue la primera que abordó el tema en un texto fundamental (Rabasa, 1997: 100).

Artículo 27. El texto de este artículo contiene una de las reivindicaciones de la Revolución: la cuestión rural. En el proyecto de Carranza, a pesar de que hacía alusión a los ejidos de los pueblos, fue considerado insuficiente por los legisladores. La reforma agraria fue abordada por su complejidad hasta las últimas sesiones. La Comisión de Constitución calificó al artículo 27 como "el más importante de todos cuantos contenga la Constitución que el $\mathrm{H}$. Congreso viene elaborando". Añadió que reconocía tres clases de derechos territoriales: el de la propiedad plena (individual o colectiva); el de la propiedad privada, restringida de las corporaciones o comunidades de la población y dueños de tierras y aguas poseídas por ejidos; y el de las posesiones

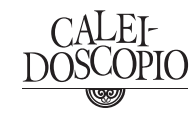


de hecho. Tras largos y encendidos debates, y con varias aportaciones de diferentes constituyentes, finalmente se completó el artículo 27 del proyecto del primer jefe que comprendió diferentes materias, pero muy especialmente la agraria (INEHRM, X: 105). ${ }^{5}$ La reforma agraria consistió en hacer que la tierra tuviera una función social para equilibrar la riqueza pública; así, cada mexicano poseería un pedazo de tierra para trabajar; conceder acción popular para denunciar los bienes que estuvieran en manos de las Iglesias; considerar a la ley agraria del 6 de enero de 1915 como ley constitucional; proscribir el latifundio y lograr la pequeña propiedad (Rabasa, 1997: 101).

Es importante señalar que, con el transcurso del tiempo, el artículo 27 ha sufrido reformas relativas al tema agrario. La más importante aconteció en el gobierno del presidente Salinas de Gortari que, en esencia, cambió el régimen estricto del ejido para hacer más productivo y capitalizar el campo. Los ejidatarios pasaron a convertirse en propietarios de su parcela (Rabasa, 1997: 101).

Artículo 130. La Revolución se alimentó de la reivindicación de las causas sociales, decidida a concluir con los privilegios de los poderosos, así, respecto a la Iglesia, estableció dos artículos: el 24, relativo a la libertad de cultos, circunscrita a los templos o a los domicilios particulares; y el 130, en el que, además de aceptar las propuestas de Carranza sobre la independencia del Estado y la Iglesia, así como otros principios de las Leyes de Reforma, los constituyentes negaron toda personalidad jurídica a las agrupaciones religiosas denominadas Iglesias, y a los ministros del culto les limitó severamente sus derechos civiles. El 28 de enero de 1992 se introdujo una reforma al artículo, impulsada también por Salinas de Gortari, donde se otorga el reconocimiento a la personalidad de las Iglesias, se amplía el ejercicio de los derechos políticos y civiles de los ministros de culto, se establece la posibilidad de que las asociaciones religiosas adquirieran bienes en propiedad para el cumplimiento de sus fines y su participación, sujeta a la aprobación de las autoridades en la impartición de la educación (Rabasa, 1997: 101).

5 Francisco J. Mújica Velázquez (3 de septiembre de 1884-12 de abril de 1954). Diputado del Congreso Constituyente de Querétaro por el $15^{\circ}$ distrito electoral, con sede en Zamora, Michoacán. Fue líder del grupo radical o jacobino (el jacobinismo busca la prohibición de cultos, supresión de la libertad de creencias y erradicación forzada de la religión) del Congreso. Asistió como presidente de la Primera Comisión de Puntos Constitucionales, participó en la elaboración de los dictámenes y en la redacción de la versión definitiva de los artículos 3ㅜ, 27 y 123.

116 CENTENARIO DE LA CONSTITUCIÓN 1917-2017 
Además de lo señalado, la Constitución de 1917 reconoció al municipio libre, dotándolo de un ayuntamiento electo popularmente, con la libre administración de su hacienda e investido de personalidad jurídica.

La Constitución de Querétaro, desde su expedición, generó reacciones de inmediato. Manuel Aguirre Berlanga reconstruyó en tono periodístico el origen de la nueva ley y los trazos básicos de su obra. En forma más de panfleto que de libro, Jorge Vera Estañol y el arzobispo de México, José Mora y del Río, sonaron la alarma por una Constitución que consideraron aberrante, tal vez herética. Embestidas más que reflexiones. En el extranjero, el texto mereció la atención pronta de Branch, Burgues, Dekelbaum, Kerr, Moses y Whelles, entre otros. No son escasos los trabajos periodísticos acerca de la Constitución que se publicaron en los años posteriores a su promulgación (Cossío y Silva-Herzog, 2017: 9-10).

\section{LA CONSTITUCIÓN SUSTANCIAL Y EL ALCANCE DEL PODER REVISOR DE LA LEY FUNDAMENTAL}

La Constitución de 1917 tuvo el reconocimiento mundial por ser el primer texto fundamental que incorporó los derechos sociales. Surge con ella el "estado social", también llamado por los países anglosajones "estado de bienestar", que enmarca la mayor participación pública en el dominio social y económico. Le siguieron en el movimiento del constitucionalismo social al Código Supremo mexicano, la Constitución rusa de 1918 y la alemana de Weimar de 1919 (Fix-Zamudio y Valencia, 1999: 516-518). Lo que se admiraba de la Carta Magna mexicana eran sus innovaciones radicales, que naturalmente recibieron la atención de quienes buscaban sentido y consecuencia en la normativa revolucionaria. La nueva filosofía de la propiedad de la tierra, las consecuencias del régimen agrario y el estatuto jurídico del subsuelo (en especial de la minería y el petróleo), así como la fisonomía del trabajo, fueron estudiados con atención (Cossío y Silva-Herzog, 2017: 10).

Sobre los procesos de revisión constitucional, se han generado debates para determinar cuál es el límite para reformar una ley fundamental. Guastini (2001: 41) señala que hay que distinguir la reforma constitucional de la instauración de un nuevo código fundamental, para ello propone dos respuestas: analizar el concepto sustancial y formal que se tenga de una constitución.

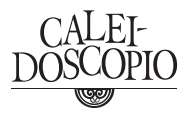


Concepto sustancial. Una Constitución es una totalidad coherente y conexa de valores ético-políticos, cuya identidad material descansa en un conjunto de principios supremos que la caracterizan y distinguen de cualquier otra Constitución. De ahí, que una cosa sea revisar la ley fundamental sin alterar la identidad material o valorativa, y otra cuestión es modificar el espíritu de la Constitución existente, esto es, alterar, perturbar o subvertir los valores ético-políticos que la caracterizan (Guastini, 2001: 42).

Concepción formal. Una Constitución no es más que un conjunto de normas. Ahora bien, un conjunto se identifica por la simple enumeración de los elementos que lo componen. Existen tres tipos posibles de reforma constitucional: i) la introducción de una norma nueva, ii) la supresión de una norma preexistente, y iii) la sustitución de una norma preexistente (Guastini, 2001, 43).

La esencia de la Constitución se expresa en la integración dentro de su texto de los principios políticos, sociales y económicos que se encuentran arraigados en el ser, el modo de ser y el querer ser de un pueblo. A estos principios esenciales se les suele designar con el nombre de "decisiones políticas fundamentales", decisiones que también son de índole social y económica (Burgoa, 1994: 380-381).

Las decisiones políticas fundamentales que preconiza la Constitución mexicana y sobre las cuales se sustenta el orden jurídico fundamental, a criterio de Ignacio Burgoa (1994: 384), son: la soberanía popular, la división de poderes, la normativización del poder público, el goce y disfrute de los derechos humanos, el juicio de amparo, la no reelección presidencial, la consagración de las garantías sociales en materia obrera y agraria, la forma republicana de gobierno, el federalismo y la democracia.

Las decisiones políticas fundamentales no son universales, están determinadas por la historia y la realidad sociopolítica de cada comunidad. Las decisiones fundamentales son parte de la historia del hombre y su anhelo de libertad. Casi todas las constituciones contienen cuatro decisiones fundamentales que son: la soberanía, la declaración de derechos humanos, la división de poderes y el sistema representativo. En México, para Carpizo (1998: 298), además se contienen otras tres: el régimen federal, el control de la constitucionalidad de leyes y actos, y la supremacía del Estado sobre las iglesias.

Enmarcada la esencia constitucional a través de sus decisiones políticas fundamentales, la cuestión recae en determinar hasta dónde 
es posible reformar una Constitución. Se han formado dos corrientes que aglutinan la mayor parte de los sistemas de revisión constitucional que han marcado los alcances y límites de la enmienda a un texto fundamental, éstas son: la alemana y francesa.

Doctrina alemana. Para esta corriente, integrada por Carl Schmitt, Hauriou, Bagehot, Guastini, Burgoa, Rabasa, entre otros, en ningún caso puede la reforma constitucional ser llevada hasta modificar los principios supremos de la Constitución existente, tales principios son límites infranqueables para la reforma constitucional. Esos principios ya estudiados están integrados por los derechos humanos, la división de poderes, la garantía jurisdiccional de la Constitución, el sistema republicano, la democracia, el federalismo, la no reelección del ejecutivo federal, la separación del Estado-Iglesia (Burgoa, 1994: 380; Guastini, 2001: 41; Tena, 1998a: 47).

Doctrina francesa. Para esta corriente, la Constitución es susceptible a ser reformada en su totalidad. Laferrière (en Tena, 1998a: 53) manifestaba en este sentido:

Desde el punto de vista jurídico, el procedimiento que consiste en decretar la inmutabilidad de una parte de la Constitución, carece de valor. El poder constituyente que se ejerce en un momento dado no es superior al poder constituyente que se ejercerá en lo porvenir, y no puede pretender restringirlo, así sea en un punto determinado. Disposiciones de este género son simples votos, manifestaciones políticas, pero no tienen ningún valor jurídico, ninguna fuerza obligatoria para los constituyentes futuros. Toda Constitución debe ser revisable en su totalidad, sin mengua de que se prevea, para alguno de los artículos, un procedimiento de revisión más complicado.

Thomas Jefferson afirmó que una periódica enmienda constitucional era parte necesaria de toda democracia que aspirara a funcionar bien: "Cada generación es tan independiente de la anterior como lo fue ésta de todas las que la precedieron. Por eso tiene, como ellas, el derecho de elegir por sí misma la forma de gobierno que considere que promoverá mejor su propia felicidad [...] Los muertos no tienen derechos" (Sunstein, 2012: 344)

Carré de Malberg, Duguit, Bryce, Munro y Tena Ramírez, entre otros, apoyan esta doctrina. Munro, en adición a lo citado, señaló: "Una constitución es manifestación de la soberanía popular, y una generación del pueblo difícilmente podría imponer, para siempre, una

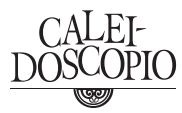


limitación a la soberanía de las futuras generaciones. Esto constituiría un gobierno de los cementerios" (Sunstein, 2012: 52).

De lo anterior, concluimos que para la doctrina alemana, las decisiones políticas fundamentales no están sujetas a la revisión constitucional; mientras que para la doctrina francesa, todo el contenido de una Constitución es reformable. México adopta el modelo de revisión constitucional francés, por lo que, atendiendo al procedimiento consignado en el artículo 135 de la Ley Fundamental, todo su texto puede ser reformado.

LA CONSTITUCIÓN MEXICANA ACTUAL: ENTRE LA REFORMA Y LA DEFORMACIÓN

La Constitución aprobada en Querétaro el 31 de enero de 1917 y la actual no es la misma. Podemos preguntarnos: ¿el proceso de revisión a la que ha sido sometida en una centuria ha dado origen a una nueva Constitución? Probablemente no. Sigue siendo esencialmente la Carta social revolucionaria, mantiene sus decisiones políticas fundamentales. La mayoría de sus reformas constitucionales se justifican por necesidades de naturaleza económica y social, para adecuarnos a las necesidades de desarrollo del proceso democrático, por ello, las múltiples reformas han sido justificadas. El problema que visualizamos consiste en que no todas están bien redactadas.

Numéricamente la Constitución no ha cambiado. Siguen existiendo únicamente 136 artículos, sólo que cada uno de ellos ha incrementado sus contenidos propios: apartados, párrafos, fracciones o incisos son hoy considerablemente más numerosos que en 1917. El incremento de las palabras en la Carta Magna es tan dramático que, a manera de ejemplo, encontramos que en 1917 el artículo 41 tenía 63 palabras; hoy tiene más de cuatro mil. La Constitución mexicana es la segunda carta fundamental con más palabras a nivel mundial (sólo es más extensa la Ley Suprema de la India) (Cossío en Fierro, 2017: 10; Salazar en Fix- Fierro y Valadés, 2017: XIX).

La Constitución mexicana, desde su expedición hasta el 15 de septiembre de 2017, ha sufrido 707 reformas a través de 232 decretos de reforma constitucional. A continuación, se presenta la Tabla 1 que ilustra su evolución por periodo de gobierno. 


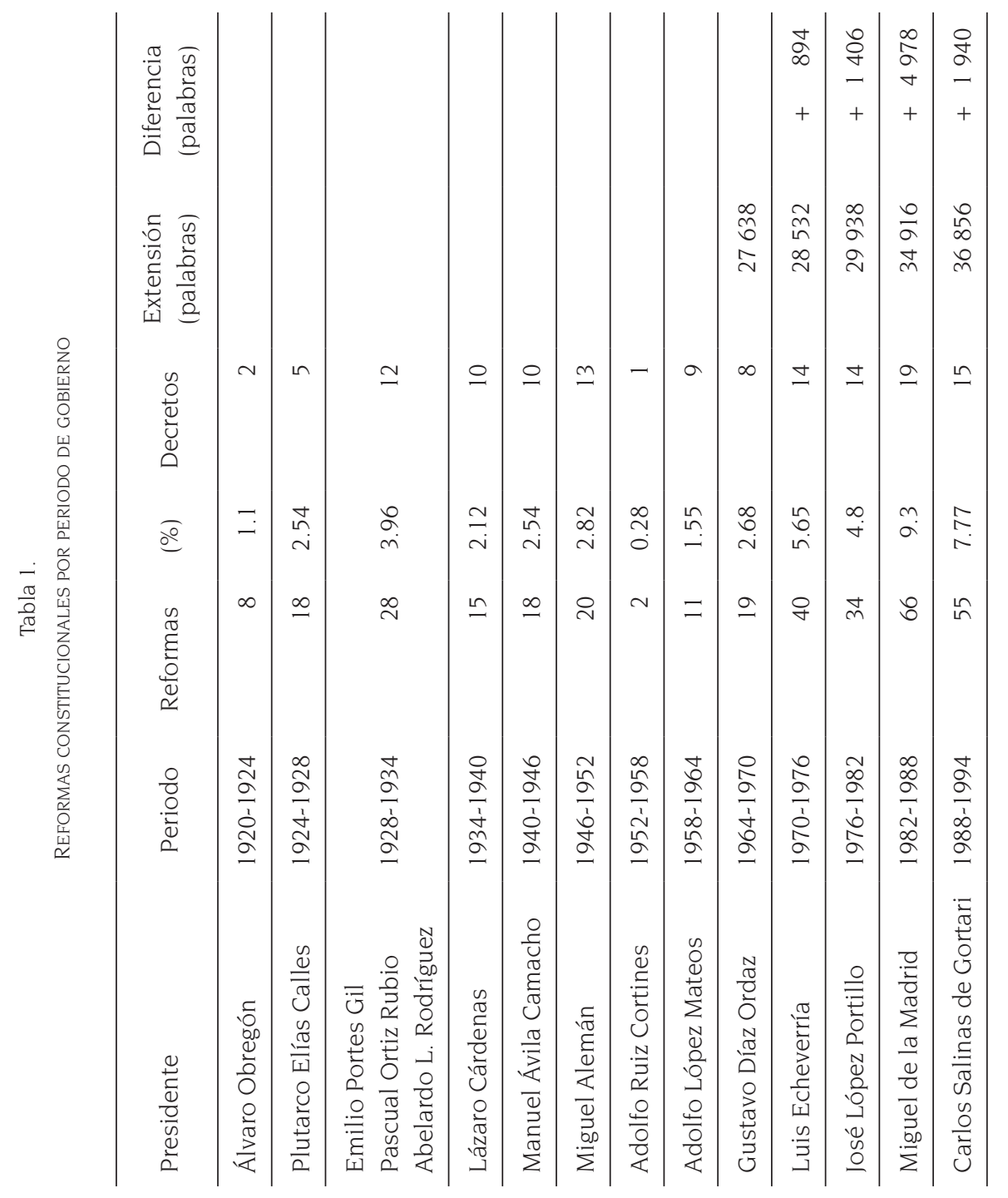




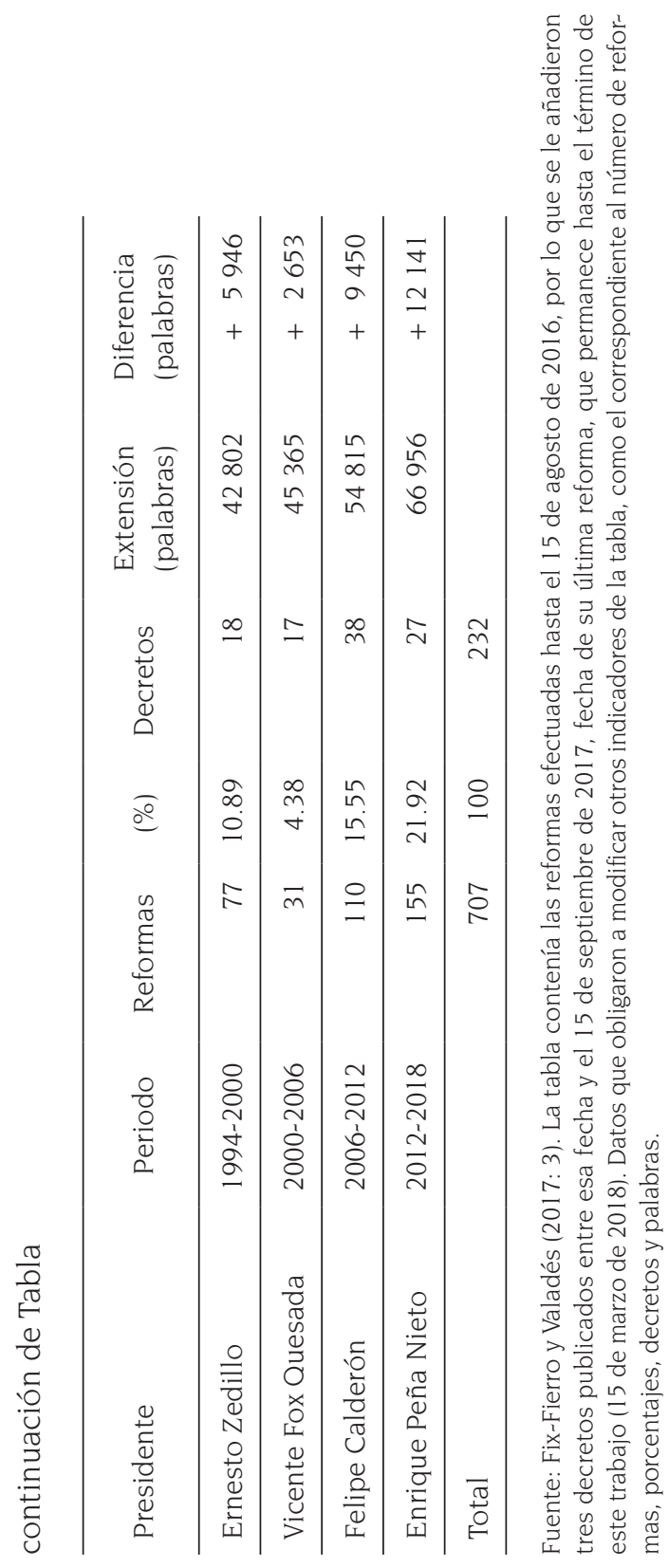

122 CENTENARIO DE LA CONSTITUCIÓN 1917-2017 
Al ser tantas las reformas y adiciones al texto fundamental, su análisis se debe sistematizar para entender bien qué ha pasado con la narrativa constitucional. El ministro José Ramón Cossío Díaz identifica tres ciclos constitucionales en la Carta Magna. ¿Qué determina un ciclo? La respuesta es la materialidad de los cambios producidos. Los ciclos identificados son los siguientes (Cossío en Fierro, 2017: 11-12):

1. Político-electoral.

2. Sobre federalismo.

3. Derechos humanos.

Hasta antes de la reforma política de 1977, poco se había modificado en la materia electoral. Los derechos y las obligaciones electorales se mantuvieron prácticamente iguales, los medios de elección fueron los mismos, la organización electoral estaba en manos de las autoridades e imperó la autocalificación. Después de ese año, las reformas electorales no han parado. Los derechos se han ampliado, los partidos tienen un estatus competente y central, las elecciones son organizadas entre autoridades y partidos, y los tribunales califican no sólo la validez de aquéllas, sino mucho de lo que acontece en el devenir electoral (Cossío en Fierro, 2017: 12).

Respecto a la distribución de competencias entre el gobierno central federal, las entidades federativas y los municipios, desde 1970 se ha efectuado un constante proceso de revisión constitucional para otorgarle mayores facultades al gobierno central federal y a los municipios en detrimento de las localidades. Los estados han disminuido sus facultades en razón de la competencia residual que le otorga el artículo 124 constitucional (Cossío en Fierro, 2017: 13).

En cuanto a los derechos humanos, el cambio inicia también en 1970 con la adición de algunos de los derechos sociales. Con la reforma del 10 de junio de 2011 y el nuevo texto del artículo $1^{\circ}$ constitucional, los derechos humanos se expanden al ampliarse los contenidos en la Ley Fundamental, y con los contenidos en los tratados internacionales celebrados por el Estado mexicano, al ser considerados como una parte extensiva de la Carta Magna (Cossío en Fierro, 2017: 13).

Durante los cien años de vigencia de la Constitución, bajo las directrices del propio texto, el órgano revisor de la norma fundamental

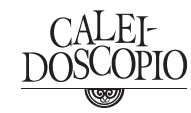


es el "Constituyente Permanente" (Tena, 1998a: 46), ${ }^{6}$ órgano establecido en el artículo 135 constitucional. En la actualidad, con las atribuciones y el papel que representa la Suprema Corte de Justicia de la Nación como máximo tribunal del país, sus facultades de interpretación de la norma fundamental le ha permitido decir lo que la Constitución significa, tal y como aconteciera con el célebre caso Marbury vs. Madison. La Suprema Corte de Justicia ha evolucionado de una corte de casación a un tribunal constitucional. Sus atribuciones desde la reforma de 1994 le otorgan la posibilidad en exclusiva de declarar la invalidez general de las normas con efectos generales hacia el futuro. Su actuación es la de un legislador positivo, a través de una función activa y creadora del derecho, cuando emite sus sentencias como tribunal constitucional (Ferrer, 2011: 59-77).

Al momento de la publicación de la Constitución centenaria, la idea jurídico-política dominante respecto a su contenido era que con él y con las interpretaciones realizadas por las autoridades nacionales, se cerraba el orden jurídico mexicano. En la actualidad, debido a la aceptación del Estado mexicano de la jurisdicción de la Corte Interamericana de Derechos Humanos (así como otros mecanismos con funciones semejantes), nuestra Constitución no es la cumbre de nuestro orden jurídico. Al menos en materia de derechos humanos, lo que diga la Corte Interamericana tiene la posibilidad de sobreponerse a lo decidido por los órganos nacionales, la Suprema Corte incluida (Cossío en Fierro, 2017: 18).

La división de poderes, como se escribió en el texto original, no tiene la misma connotación. Hoy, con la introducción de los llamados órganos constitucionales autónomos, se ha trastocado el principio. Diego Valadés ha encabezado la crítica por la creación de numerosos órganos constitucionales autónomos, nacidos como parte de las reformas constitucionales suscitadas entre los años 2013 a 2015, impulsadas por el Presidente de la República y los grupos parlamentarios de los tres partidos políticos principales en el Congreso de la Unión

6 El ministro Felipe Tena Ramírez nombró al poder revisor de la Constitución bajo el término de Constituyente Permanente, del que señaló: "Este órgano tiene que participar, en alguna forma, de la función soberana, desde el momento en que puede afectar la obra que es expresión de la soberanía (la Constitución). Su función es, pues, constituyente; y como, por otra parte, se trata de un órgano que sobrevive al autor de la Constitución, cuya vida se extinguió con su tarea, consideramos que merece, por todo ello, el nombre de Poder Constituyente Permanente". 
(PRI, PAN y PRD). En una entrevista efectuada a Diego Valadés sobre estos órganos, manifestó lo siguiente:

[...] Un buen sistema democrático se construye esencialmente con un sistema de partidos y un sistema representativo, porque es con el que estamos en contacto los ciudadanos y con un gobierno responsable ante ese sistema representativo; de manera que sean los representantes de la nación los que puedan estar convocando a quienes ejercen las funciones de poder para que informen, para que reciban los puntos de vista y orientaciones que les dan los representantes de la nación y eventualmente, si los informes y el desempeño no resulta satisfactorio, los representantes de la nación puedan censurarlos. Digamos, esto es lo que pasa en términos muy concretos, muy sintéticos, en un sistema democrático que funcione razonablemente, pero que está pasando en este caso que tú señalas y que es clave; estamos construyendo ínsulas de poder que ya no dependen ni del gobierno, cuyo titular todos elegimos, ni del sistema representativo que, de acuerdo con la Constitución, es eso, la representación de la nación, y entonces estamos construyendo ínsulas de poder autónomas y realmente soberanas porque no responden ante nadie, y ahí estamos dándole facultades en materia de comunicaciones, telecomunicaciones, en materia de energéticos, en materia financiera, en una pluralidad de cuestiones que son del interés de la sociedad mexicana y cuyos integrantes de esos organismos no van a responder por lo que hagan ni ante el gobierno, ni ante el congreso. Bueno esto es un, en el orden, calificándolo en términos muy coloquiales, jes un disparate! [...] la presencia creciente de este tipo de organismos refleja, repito, desconfianza, no es la lógica de las agencias gubernamentales que existen en Europa o que existen en Estados Unidos, donde la razón ha sido simplemente construir organismos especializados técnicamente, eficaces, muy profesionales, pero todos son responsables ante los parlamentos o ante el Congreso de los Estados Unidos; o si estamos en Europa, esos organismos son responsables ante el Parlamento europeo, si son órganos europeos, o ante el Parlamento británico, o al Parlamento alemán, y en Estados Unidos ante el congreso, y rinden cuentas muchas veces semanalmente. En México no, son agencias técnicas a las que les hemos dado todo el poder político, esto es una verdadera anomalía en cualquier sistema constitucional, por eso lo califiqué como un disparate $[\ldots]$ se ha ido desmembrando el poder, y se han ido construyendo ínsulas que, pues en cuanto a su desempeño, tendremos que confiar ya en lo que cada uno de los integrantes y el conjunto de los integrantes

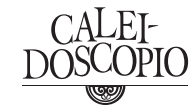


de esos órganos quieran responder a su propia conciencia, porque ellos no son responsables más que a su conciencia, lo cual es gravísimo, como si estuviéramos en la edad media en materia de poder [... ¿Cuándo hemos visto a ese funcionario? (el gobernador del Banco de México), o a otros funcionarios de organismos equivalentes, que asistan ante las cámaras del congreso para informar ahí de las decisiones que están haciendo, si nosotros vemos qué hacía el señor Bernal o qué hacían sus sucesores o antecesores: iban permanentemente ante las comisiones del Senado o de la cámara de representantes de los Estados Unidos a informar de lo que estaba ocurriendo. Yo recuerdo, hasta como anécdota, que cuando se dio la epidemia terrible hace unos años de H1N1, estábamos mejor informados en México por lo que decía la Secretaría de Salud (Kathleen Sebelius), que informaba todos los días a las comisiones de ambas cámaras en Estados Unidos, acerca de la evolución de la epidemia aquí en México, que por las autoridades mexicanas, porque las autoridades mexicanas jamás fueron a la cámara de diputados a informar. Es una verdadera paradoja que a veces nos informemos de lo que pasa en México a través de gobiernos extranjeros, en este caso, ocurrió [...] cuando hablamos ya de estos organismos autónomos, que se le llama organismos constitucionales autónomos, que no forman parte de ningún poder, están rompiendo inclusive un principio que también es arcaico, el principio de la división de poderes, también un principio que muchas constituciones han abandonado, pero que en México también rompimos; así como te decía hace un momento en la contradicción de la naturaleza plural del congreso y la naturaleza unitaria del gobierno: hay una contradicción entre una Constitución que nos dice que hay tres órganos del poder y que formalmente, además de esos tres órganos que ya construyó la propia Constitución, hay otra decena, como tú señalas, así que la propia Constitución se contradice internamente y esto, por supuesto, en desdoro desea norma, lo cual no nos conviene a los mexicanos, no nos conviene tener una Constitución desprestigiada (Fuentes, 2014).

La Constitución no es y nunca fue un texto infranqueable, sino un texto abierto a diferentes circunstancias, actores y tiempos; pero también es necesario tomar en consideración que otro tanto de enmiendas ha sido establecido por la desconfianza entre los interlocutores políticos, resultando la introducción de elementos reglamentarios en la norma suprema, situación que la ha desfigurado no en el orden de su contenido, sino en el orden de su redacción. Por lo mismo, con 
independencia de las reformas que se hacen necesarias para profundizar y complementar el proceso democrático del país, tenemos el reto de construir una democracia institucional que seguirá requiriendo la transformación constitucional. Por eso, es pertinente ver a la Constitución como un objeto de estudio por sí mismo y necesitamos reescribirla, más que pensar en crear un nuevo orden constitucional. La meta es regresar a la coherencia sistemática y funcional del texto original.

\section{CONCLUSIONES}

1. ¿Se requiere una nueva Constitución? Ante la cuestión creemos que México no requiere elaborar una nueva Ley Fundamental, siguiendo a Sartori, Fix, Valadés y Salazar, entre otros, creemos que la Constitución revolucionaria de 1917 es una buena Constitución, su texto original contenía principios y derechos innovadores y apropiados, que aún hoy consideramos de actualidad. Esto nos lleva a pensar que una nueva Constitución no es lo que requiere el país para su mejor funcionamiento. Apoyamos la idea del profesor de Columbia que decía: "nunca lo conseguirán (crear una nueva norma suprema), y si lo consiguen, será horrible". Es importante recobrar lo que se admiraba de la Carta Magna mexicana en 1917: sus innovaciones radicales, que naturalmente recibieron la atención de quienes buscaban sentido y consecuencia en la normativa revolucionaria. La nueva filosofía de la propiedad de la tierra, las consecuencias del régimen agrario y el estatuto jurídico del subsuelo (en especial de la minería y el petróleo), así como la fisonomía del trabajo, aspectos que fueron estudiados con atención. Fue tan importante el contenido de la Constitución social mexicana que por ello, en 1918, la Carta rusa y la alemana de Weimar, en 1919, la siguieron.

2. El consenso que se ha vislumbrado por parte de los estudiosos de la Constitución es que se requiere emprender un proyecto de reformas que se circunscriban a reescribir el texto fundamental para reordenarlo, para volver a darle una presentación sistemática y funcional como corresponde a la norma suprema, para eliminar las contradicciones, superar sus lagunas, mejorar su redacción, y

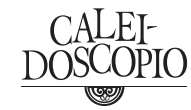


hacer una recapitulación con relación al texto. La idea es entregarle al país y a la sociedad una Constitución legible.

3. El México de la Revolución, lleno de contrastes y necesitado de obtener justicia social, vio en la lucha armada la necesidad de refundar al Estado. En los planes y proclamas revolucionarios encontramos el germen de lo que hoy reconocemos como los principios político-fundamentales que le dan sentido y esencia a la Constitución. Cuando Carranza expidió las reformas al Plan de Guadalupe publicadas el 14 de septiembre de 1916, no tenía del todo claro la consecuencia de reunir una asamblea constituyente, todo indica que su interés estaba más alineado a congregar una asamblea legislativa que adoptara facultades de poder revisor de la Constitución, para elevar a rango constitucional las leyes expedidas por los constitucionalistas en 1915; así como integrar el programa propuesto en su Plan de Guadalupe y otros planes y proclamas revolucionarios en el texto fundamental, con el propósito de ganarse a la ciudadanía en demérito de sus opositores Villa y Zapata.

4. Uno de los más graves errores que cometió Venustiano Carranza en la convocatoria al Congreso Constituyente de Querétaro, fue prohibir en el artículo $4^{\circ}$ del decreto que reforma el Plan de Guadalupe, la elección de los hombres que ayudaron con las armas o en los empleos públicos a los gobiernos y facciones hostiles a los constitucionalistas dirigidos por Carranza, lo que hizo que el Congreso fuera ilegítimo de origen, poniendo en riesgo toda su obra, que fue y es hoy nuestra Constitución.

5. El problema de la ilegitimidad de la Constitución revolucionaria fue estudiado y solventado por la doctrina, señalando que la Constitución fue obra de una asamblea constituyente ad hoc, producto de una verdadera revolución, que por serlo modifica en forma violenta los fundamentos constitucionales de un Estado, redactando un nuevo Código Supremo, que es un producto extra ordinem, privado de fundamento de validez en normas propias del ordenamiento constitucional precedente.

6. La idea de reforma a la Constitución liberal de 1857 que tenía Carranza fue rechazada por los constituyentes que plantearon crear un nuevo texto fundamental que refundara al Estado mexicano, incluyendo la anhelada reforma agraria y social. De acuerdo con esta interpretación, a Carranza no le correspondía el papel de 
líder restaurador de las libertades y derechos de los ciudadanos mexicanos, sino el de viejo reaccionario que se opuso a las reformas que exigía la Revolución.

7. Las decisiones políticas fundamentales que preconiza la Constitución mexicana y sobre las cuales se sustenta el orden jurídico fundamental son: la soberanía popular, la división de poderes, la normativización del poder público, el goce y disfrute de los derechos humanos, el juicio de amparo, la no reelección presidencial, la consagración de las garantías sociales en materia obrera y agraria, la forma republicana de gobierno, el federalismo, la democracia y la separación del Estado-Iglesia.

8. A la pregunta de ¿hasta dónde podemos reformar la Constitución? Encontramos como respuesta dos doctrinas: la doctrina alemana y la doctrina francesa. Para la doctrina alemana, las decisiones políticas fundamentales no pueden ser tocadas en la revisión constitucional; mientras que para la doctrina francesa todo el contenido de una Constitución es reformable. México adopta el modelo de revisión constitucional francés, por lo que atendiendo al procedimiento consignado en el artículo 135 de la Ley Fundamental, todo su texto puede ser reformado.

9. Thomas Jefferson afirmó que una periódica enmienda constitucional era parte necesaria de toda democracia que aspirara a funcionar bien: "Cada generación es tan independiente de la anterior como lo fue ésta de todas las que la precedieron. Por eso tiene, como ellas, el derecho de elegir por sí misma la forma de gobierno que considere que promoverá mejor su propia felicidad [... ]. Los muertos no tienen derechos".

10. Se cuestiona si las reformas efectuadas al texto fundamental en 100 años han producido una nueva Constitución. Consideramos que no, es claro que la Ley Fundamental aprobada en Querétaro el 31 de enero de 1917 y la actual no son la misma. Desde su expedición hasta el 15 de septiembre de 2017, la Constitución mexicana ha sufrido 707 reformas a través de 232 decretos de reforma constitucional. ¿Podemos decir que el proceso de revisión a la que ha sido sometida en una centuria ha dado origen a una nueva Constitución? Probablemente no. Consideramos que la Constitución Política de los Estados Unidos Mexicanos sigue siendo esencialmente la Carta social revolucionaria, ya que mantiene sus decisiones políticas fundamentales. La mayoría de

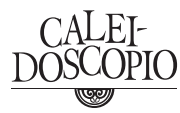


sus reformas constitucionales se justifican por necesidades de naturaleza económica y social para adecuarnos a las necesidades de desarrollo del proceso democrático, por ello, las múltiples reformas han sido justificadas. El problema que presenta la Constitución consiste en que no todas las reformas introducidas son buenas, como el caso del exceso de órganos constitucionales autónomos introducidos, que han restado centralidad y fortaleza al Poder Ejecutivo; así como el hecho de que una buena parte de esas reformas no están bien redactadas.

11. La introducción de elementos reglamentarios en el texto de la norma constitucional ha terminado por deformar la Constitución, no en el orden de su contenido, sino en el orden de su redacción. La Ley Fundamental requiere ser reescrita para darle coherencia y bajar del texto el exceso reglamentario introducido, para trasladarlo hacia las leyes reglamentarias, orgánicas y ordinarias federales encargadas de desplegar los contenidos programados en la Constitución.

\section{REFERENCIAS}

Andrews, C. (2017). De Cádiz a Querétaro. Historiografía y bibliografía del constitucionalismo mexicano. $1^{\text {a }}$ ed., México: FCE/CIDE.

Burgoa, I. (1994). Derecho constitucional mexicano. $9^{a}$ ed., México: Porrúa.

Carpizo, J. (1998). Estudios constitucionales. $6^{\text {a }}$ ed., México: UnAm/Porrúa.

Cossío Díaz, J. R. y Silva-Herzog Márquez, J. (2017). Lecturas de la Constitución. El constitucionalismo mexicano frente a la Constitución de 1917. $1^{a}$ ed., México: FCE.

Elster, J. y Slagstad, R. (Coords.). (2012). Constitucionalismo y democracia. $2^{\mathrm{a}}$ reimps., México: FCE.

Ferrer Mac-Gregor, E. (2011). La Suprema Corte de Justicia de México como legislador positivo. Anuario de Derecho Constitucional Latinoamericano, Año XVII. Uruguay, Konrad Adenauer Stiftung.

Fierro, A. E. (2017). Responsabilidad de los servidores públicos. Del castigo a la confianza. $1^{a}$ ed., México: FCE.

Fioravanti, M. (2003). Los derechos fundamentales. Apuntes de historia de las constituciones. 4a. Ed. Trad. de Manuel Martínez Neira. Madrid: Trotta. 
Fix-Fierro, H. y Valadés, D. (2017). Constitución Política de los Estados Unidos Mexicanos. Texto reordenado y consolidado. $2^{\mathrm{a}}$ ed., México: UnAm/Senado de la República/Instituto de Investigaciones Jurídicas.

Fix-Zamudio, H. y Valencia Carmona, S. (1999). Derecho constitucional mexicano y comparado. México: Porrúa.

Galeana, P. (2016). Diccionario biográfico de los diputados constituyentes de 1917. $1^{a}$ ed., México: Secretaría de Cultura/Instituto Nacional de Estudios Históricos de las Revoluciones de México.

Garciadiego, J. y Kuntz Ficker, S. (2011). La revolución armada. Nueva Historia General de México. $1^{a}$ reimps., México: El Colegio de México.

González Oropeza, M. (1998). Homenaje al Congreso Constituyente de Querétaro. $1^{\text {a }}$ ed., México: UNAm/Instituto de Investigaciones Jurídicas.

Guastini, R. (2001). Estudios de Teoría Constitucional. 1ª ed., México: Fontamara.

Instituto Nacional de Estudios Históricos de las Revoluciones de México. (2016). México:INEHRM. Diccionario biográfico de los diputados constituyentes de 1917. Siglo XXI.

Instituto NAcional de Estadística y Geografía (1910). Tercer Censo de Población de los Estados Unidos Mexicanos. En http://www.beta. inegi.org.mx/programas/ccpv/1910/.

López Barrón, X. (24/ene/2017). México necesita una nueva Constitución, pero no hay condiciones idóneas para su elaboración. Enfoque noticias [En línea]. Disponible en: http://www.enfoquenoticias.com.mx/noticias/m-xico-necesita-una-nueva-constituci-n-pero-no-hay-condiciones-id-neas-para-su-elaboraci-n.

Mora-Donato, C. (2002). El valor de la Constitución normativa. $1^{\text {a }}$ ed., México: UNAM/Instituto de Investigaciones Jurídicas.

Rabasa, E. O. (1997). Historia de las Constituciones Mexicanas. $1^{\text {a }}$ reimps., México: Instituto de Investigaciones Jurídicas.

Sunstein, C. R. (2012). Constituciones y democracias: epílogo. En Jon Elster y Rune Slagstad (Coords.). Constitucionalismo y democracia. $2^{\text {a }}$ reimps., México: FCE.

Tena Ramírez, F. (1998a). Derecho Constitucional Mexicano. 32ª ed., México: Porrúa.

(1998b). Leyes Fundamentales de México 1808-1998. $21^{\text {a }}$ ed., México: Porrúa. 


\section{ELECTRÓNICAS}

Fuentes, M. L. (4/sep/2014). Entrevista a Diego Valadés. México Social. La Constitución mexicana. La historia y la perspectiva. Canal 11. Disponible en: https://www.youtube.com/watch?v=HMcWid4RmSk.

Instituto Nacional de Estadística y Geografía (INEGI). (1910). Tercer Censo de Población de los Estados Unidos Mexicanos 1910. Disponible en: http://www.beta.inegi.org.mx/proyectos/ccpv/1910/. Islas, J. (2/mayo/2012). Entrevista a Giovanni Sartori. Reflexiones sobre la democracia en México. Canal 22. Disponible en: https://www.youtube. $\mathrm{com} /$ watch?v=6UtrlOx4RTw.

\section{SOBRE EL AUTOR}

Claudio Antonio Granados Macías (claudiogranados72@hotmail.com) es doctor en Derecho por la Universidad de Guadalajara, doctor en Ciencias Sociales y Humanidades, maestro y licenciado en Derecho por Universidad Autónoma de Aguascalientes. Es profesor investigador del Departamento de Derecho de la UAA (ORCID: 0003-4327-272X). Sis 